\section{Reunión Consultiva Regional de las Américas sobre la Evaluación del Desempeño de los Sistemas de Salud ${ }^{1}$}

Palabras clave: sistemas de salud, evaluación de sistemas de salud, Organización Mundial de la Salud.

\footnotetext{
1 Basado en "Critical issues in health system performance assessment", documento que resume los temas abordados en la Reunión Consultiva Regional de las Américas sobre la Evaluación del Desempeño de los Sistemas de Salud, Washington, D.C., 8 a 10 de mayo de 2001. El documento completo se encuentra en el siguiente sitio de la Web: www.paho.org/English/HSP/HSO/hspmtg_eng.htm
}

En su Informe sobre la salud en el mundo 2000 (ISM 2000), la Organización Mundial de la Salud, basándose en los datos reunidos por un grupo de consulta, enumeró una serie de medidas que se consideran necesarias para mejorar el desempeño de los sistemas de salud. Lamentablemente, una serie de factores de difícil corrección obstaculizó la labor analítica del grupo. A la escasez de datos básicos, en algunos casos contradictorios y en otros poco comparables, se sumó la falta de un consenso en torno a las metas y objetivos de los sistemas de salud de los distintos países. Por otra parte, los análisis de desempeño efectuados previamente se habían basado, la mayor parte de las veces, en la evaluación de procesos y no de resultados. De ahí que en el ISM 2000 se intentara crear un marco conceptual, aplicable en todas partes, para la evaluación adecuada y uniforme de los sistemas de salud.

La emisión del ISM 2000 despertó un intenso debate mundial sobre la idoneidad de los procesos empleados para su elaboración. Ante las fuertes críticas de los Estados Miembros, la Directora General de la OMS anunció su intención de tomar las siguientes medidas antes del próximo informe, que será emitido por la OMS en octubre de 2002:

- Establecer un proceso de consulta con la participación de expertos y funcionarios públicos de todos los Estados Miembros

- Preguntar a cada Estado Miembro qué datos son los más apropiados para evaluar el desempeño de su sistema sanitario, y revelar a cada uno los valores de los indicadores obtenidos a partir de esos datos

- Proporcionar a cada Estado Miembro una copia preliminar del ISM antes de su diseminación

- Formar un grupo de consulta, integrado por miembros del Comité Ejecutivo y del Comité Asesor sobre Investigaciones de Salud, para monitorear el apoyo que brinda la OMS a la evaluación de los sistemas sanitarios

Como consecuencia de estas decisiones, del 8 al 10 de mayo de 2001 se celebró en Washington, D.C., en la Sede de la Organización Panamericana de la Salud (OPS), la Reunión Consultiva Regional de las Américas sobre la Evaluación del Desempeño de los Sistemas de Salud, cuyos objetivos fueron los siguientes, entre otros: a) examinar distintos 
enfoques conceptuales y metodológicos para evaluar el desempeño de dichos sistemas; b) explorar las experiencias de distintos países de la Región en torno a la evaluación de sus sistemas sanitarios; c) identificar los aspectos indispensables de un esquema universal para medir con regularidad el desempeño de los sistemas de salud y rendirle un informe periódico a la OMS; d) examinar el vínculo entre los métodos usados para evaluar el desempeño de los sistemas de salud y los procesos de definición de políticas y de toma de decisiones en los niveles gerenciales; e) elaborar un plan de trabajo para la cooperación internacional en favor de las iniciativas nacionales para evaluar los sistemas sanitarios.

La Reunión tuvo la participación de representantes de Argentina, Barbados, Bolivia, Brasil, Canadá, Chile, Colombia, Costa Rica, Ecuador, El Salvador, Estados Unidos de América, Jamaica, México, Nicaragua, Paraguay, Puerto Rico, Reino Unido, Uruguay y Venezuela, así como del Banco Mundial, el Convenio Hipólito Unanue, el Mercado Común del Caribe (CARICOM), la OMS y la OPS.

A continuación se resumen los aspectos sobresalientes de la discusión que suscitó en nuestra Región el ISM 2000, haciendo hincapié en tres aspectos en particular: 1) elementos conceptuales y metodológicos, incluido el uso de ciertos indicadores, 2) modificaciones del esquema de evaluación en años futuros, y 3) políticas y cuestiones gerenciales.

\section{ASPECTOS CONCEPTUALES Y METODOLÓGICOS DE LA EVALUACIÓN}

\section{¿Qué se entiende por sistema de salud?}

En el transcurso del diálogo en torno al ISM 2000 se debatieron distintas nociones de lo que constituye un sistema de salud. En su significado más estrecho, este se reduce a las actividades bajo el control directo del Ministerio de Salud, quedando excluidas muchas otras inciativas públicas o privadas ajenas a los ministerios de salud.

De acuerdo con otra definición, un sistema de salud abarca los servicios médicos individuales y los servicios de salud dirigidos a la colectividad, pero no las acciones intersectoriales destinadas a mejorar la salud. Se considerarían parte del sistema las intervenciones de salud pública tradicionales - por ejemplo, el rociamiento de viviendas con plagucidas, la diseminación de información pública-, pero no las iniciativas intersectoriales de carácter ambiental, como el saneamiento y abastecimiento de agua potable.
Una tercera definición considera como parte del sistema sanitario cualquier medida, sea o no intersectorial, cuyo fin primordial sea mejorar la salud. Según la cuarta y última definición, el sistema sanitario comprende toda medida que directa o indirectamente contribuye a mejorar la salud.

La elección de una de estas definiciones está inexorablemente vinculada con el concepto de responsabilidad. Si se opina que quienes dirigen los sistemas de salud deben ser responsables de garantizar la provisión de servicios de salud personales y colectivos de carácter esencial, así como de fomentar actividades intersectoriales para mejorar la salud, la tercera definición es la más apropiada. Su aplicación llevaría a los diseñadores de políticas a pensar en términos colectivos, más allá de los servicios médicos individuales, y a impulsar medidas para beneficiar a la población en su totalidad (procurando, p. ej., eliminar el tabaquismo, reducir los accidentes de vehículos motorizados y mejorar la dieta común). En cambio, si se considera que los directores del sistema sanitario no tienen más función que la de cerciorarse del uso adecuado de los recursos bajo su control directo, cabe usar una definición más estrecha de lo que constituye un sistema de salud.

\section{La atribución de causalidad}

Muchas medidas, estén o no directamente encaminadas a mejorar la salud, influyen sobre esta última de manera favorable o desfavorable y es preciso disponer de medios para separar la influencia que ejercen las acciones del sistema de salud de las que ejercen estas otras medidas. Parece haber dos enfoques al respecto. El primero consiste en definir indicadores parciales del grado en que se logran las metas generales de un sistema, basados en una serie de hipótesis sobre causalidad. De este modo, solamente los resultados directamente determinados por las actividades del Ministerio de Salud o de quienes definen las políticas sanitarias se miden y monitorean como parte de la evaluación del desempeño del sistema de salud.

En el segundo enfoque, la medición de los resultados se hace independientemente de la evaluación de los factores a los que dichos resultados son atribuibles, aunque dichos factores no sean obra del sistema de salud. La medida en que este último ha contribuido a reducir la mortalidad materna, por poner un ejemplo, sería evaluada como parte de la evaluación de todos los posibles factores determinantes, lo cual implica poner a prueba un número ilimitado de hipótesis mediante análisis estadísticos multivariantes. 


\section{Factores mediadores entre la provisión de servicios y una mejor salud}

Resulta evidente, sin embargo, que la provisión de un servicio médico o sanitario o la adopción de medidas intersectoriales no necesariamente se traduce en un mejor estado de salud para la población. Ello exige que haya ciertas condiciones (llamémosles factores mediadores) cuya identificación y medición han sido ampliamente debatidas. Entre ellas cabe tener en cuenta la accesibilidad física y económica de los servicios, su efectividad y su calidad. Asimismo, hay que identificar ciertas intervenciones clave (o condiciones de importancia crítica) cuya cobertura deberá medirse invariablemente.

Dada la importancia de estas intervenciones críticas como mediadores de los efectos de los servicios de salud individuales y colectivos, la OMS se propone incorporar en la evaluación del desempeño de los sistemas de salud alguna forma de monitorear la cobertura de tales intervenciones. Ello implica, a su vez, vencer ciertas dificultades técnicas si el monitoreo ha de ser válido y confiable, así como apto para ser objeto de comparación. Una dificultad radica en cómo incorporar a los proveedores del sector privado en la evaluación de la cobertura del sistema sanitario; la otra, en validar la cobertura implícita en los datos sobre la provisión de servicios. Un tercer problema está dado por las variaciones que muestra la cobertura en distintas zonas de un mismo país y en los distintos subsectores de un sistema sanitario segmentado (el sector privado, el estatal, el seguro social y demás).

\section{El desempeño y el factor del tiempo}

La eficiencia (palabra que se usó como equivalente de desempeño en todo el ISM) se define como el grado máximo en que el sistema de salud contribuye al logro de las metas sociales establecidas, teniendo en cuenta los recursos existentes dentro del sistema de salud y fuera de él. En torno a cómo definir el desempeño han surgido dos conceptos distintos. Para algunos, el desempeño es todo lo que el Ministerio de Salud puede alcanzar con los recursos que tiene a su disposición en el año en curso. Este enfoque, que es afín con la definición estrecha de un sistema de salud, no les ofrece a los definidores de políticas ningún incentivo para diseñar acciones de amplio alcance en pro de la salud. Para otros, el desempeño está dado por todo lo que se podría haber alcanzado con los recursos existentes si la combinación de políticas hubiese sido óptima. Se trata de un resultdo máximo que, aunque no se alcance en el año en curso, podría al- canzarse mediante el uso óptimo de los recursos a disposición y la adopción de buenas políticas y programas.

En el primero de los enfoques citados, el tiempo reviste una importancia decisiva, ya que algunos de los logros del presente son el resultado de las acciones del pasado. Adicionalmente, algunas de las acciones que se toman en el presente, como la adopción de programas para reducir el tabaquismo, no surtirán el efecto deseado hasta pasados varios años. El tiempo tiene menos importancia en el segundo enfoque, ya que la meta es indicar lo que podría haberse logrado en general e incentivar a los funcionarios a tener una visión de largo alcance. Técnicamente, ambos enfoques son factibles. La elección depende, en parte, de la finalidad que tenga el análisis del desempeño del sistema de salud. Si dicha finalidad es determinar si el Ministerio de Salud tuvo un buen desempeño este año, el primer enfoque es el adecuado. En cambio, si la finalidad es indicar lo que se habría podido lograr si los recursos se hubiesen usado eficientemente, tanto dentro como fuera del sistema de salud, se aplica el segundo enfoque, que mueve a los definidores de políticas a ver más allá de la provisión de servicios médicos individuales.

\section{Alcance de la noción de desempeño}

Como se ha señalado anteriormete, la palabra desempeño se usó en todo el ISM como equivalente de eficiencia, entendiéndose por esta última lo que ya hemos explicado. En sentido general, y ciertamente en las publicaciones sobre la calidad de la atención, el desempeño comprende mucho más, es decir, toda una gama de actividades en torno al uso de pruebas científicas para lograr los mejores resultados posibles o el mayor número de metas. Esto da pie a un interrogante: ¿debe la OMS modificar su terminología para adaptarla a esta definición más amplia? Si así fuese, la "evaluación del desempeño de los sistemas de salud" tendría que abarcar las siguientes actividades:

- la medición del grado en que se logran las metas;

- la medición de los recursos pertenecientes al sistema de salud y a otros sectores que se pueden usar para alcanzar estos resultados;

- el cálculo de la eficiencia con que los recursos se utilizan para lograr estos resultados;

- la evaluación de cómo las funciones del sistema influyen en los niveles observados de productividad y eficiencia;

- el diseño y la adopción de políticas para mejorar la productividad y la eficiencia. 


\section{Ponderaciones aplicables en todos los países}

En el ISM se combinaron cinco indicadores de productividad en un solo puntaje compuesto. Las mejores pruebas que había en aquel momento parecían indicar que el peso que los encuestados otorgaban a los distintos indicadores no difería de manera apreciable en los distintos grupos de encuestados. Posteriormente se ha debatido si todos los países otorgan el mismo peso a los indicadores o si muestran preferencias similares. Para poder comparar la productividad en distintos países, es necesario contar con un sistema de ponderación uniforme, aunque si los pesos varían o no se puede investigar de manera empírica.

Si los pesos varían notablemente de un país a otro, se pueden usar dos tipos de sistemas de ponderación para determinar la productividad y el desempeño generales. En un caso se aplicaría el promedio de todos los países; en el otro, se utilizarían los distintos pesos observados. De este modo se podría evaluar hasta qué punto los cálculos de desempeño y eficiencia se vieron afectados por las variaciones ponderales entre países. Si existe una gran variación se podrá determinar a partir de las diversas encuestas que se están llevando a cabo este año en muchos países, en las cuales se pregunta qué importancia se otorga a diferentes metas.

\section{Metas que aportan un beneficio social}

¿Son apropiadas las metas definidas en el ISM, a saber, salud, responsabilidad y financiamiento equitativo? Resulta evidente que el estado de salud de una población no está determinado exclusivamente por las actividades del sistema sanitario, a no ser que la definición de este último sea la más amplia de las cuatro que hemos descrito. La salud es una meta social que también obedece a la influencia de muchos otros factores, y el sistema de salud contribuye al logro de esa meta. Nadie ha puesto en tela de juicio el hecho de que el sistema existe exclusivamente para mejorar la salud poblacional, aunque muchos sostienen que el sistema debe también contribuir a otras metas sociales ajenas al estado de salud. En este contexto se suele hablar del concepto de "atención a conciencia", que abarca los ocho elementos habitualmente asociados con la idea de "responsabilidad": dignidad, autonomía, confidencialidad, atención oportuna, calidad de ciertas necesidades básicas, acceso a redes de apoyo social y capacidad para elegir al proveedor de servicios. Algunos argumentan que esta definición omite aspectos importantes de una "atención a conciencia" y en torno a este tema próximamente se llevará a cabo una consultoría técnica.
Hasta la fecha, la mayoría de las entidades encuestadas han manifestado su apoyo a medidas que garanticen la equidad en el financiamiento del sistema sanitario, entre ellas los aportes monetarios progresivos al mismo, así como su oposición a sistemas que colocan a las personas en riesgo de sufrir pérdidas económicas catastróficas como resultado de una enfermedad. No obstante, algunos dudan de la idoneidad social de este enfoque en sus países; otros se preguntan si el indicador de equidad financiera utilizado en el ISM refleja en realidad la preocupación pública por las contribuciones financieras progresivas.

Hay quienes sostienen que el sistema de salud contribuye a una variedad de metas sociales además de las que indica el ISM. El crecimiento económico y una mayor participación social se ofrecen como ejemplos en este contexto. Pero si estas metas han de incluirse en la evaluación periódica del desempeño del sistema de salud dependerá, en parte, de la medida en que el sistema contribuya a ellas.

\section{Implicaciones para las políticas sanitarias}

Por último, cabe señalar que la evaluación del desempeño de los sistemas de salud no es una meta en sí misma; cobra sentido solamente porque arroja las pruebas científicas necesarias para adoptar políticas, programas y estrategias de mayor utilidad. Han surgido varias sugerencias en torno a la manera de estrechar el vínculo entre la evaluación del desempeño y el diseño de políticas dirigidas a mejorarlo, lo cual exige incrementar los esfuerzos por fortalecer ciertas funciones del sistema, como el financiamiento, la generación de recursos, la provisión y la dirección. Actualmente no se dispone de indicadores de la contribución de cada una de estas funciones al desempeño total, y es necesario que los diseñadores de políticas cuenten con mecanismos para monitorear estas funciones y calcular su impacto individual sobre el desempeño y la eficiencia generales del sistema. Con el fin de diseñar mecanismos de este tipo, se han planificado diversas consultorías que se celebrarán en el futuro próximo. Asimismo, los funcionarios que definen las políticas necesitan disponer de mecanismos para separar la contribución individual de cada componente del sistema de salud (p. ej., las acciones intersectoriales y los servicios médicos personales) al desempeño total, a fin de saber si deben transferir recursos de un componente a otro. Esto puede lograrse, por ejemplo, si se fortalece el vínculo entre la evaluación del desempeño y los análisis de efectividad en función del costo, iniciativa que está actualmente en marcha en la OMS. 


\section{FUTURAS MODIFICACIONES DEL ESQUEMA DE EVALUACIÓN}

\section{La necesidad de medir funciones y adelantos}

Se desprende de todo lo antedicho que quizá sea más útil contemplar toda una gama de actividades bajo la evaluación del desempeño, que mirar el desempeño como si fuese sinónimo de eficiencia. De ese modo se pueden identificar las áreas que plantean dificultades y que requieren atención especial, con lo que se facilita la adjudicación de recursos. El concepto más amplio de lo que consituye el desempeño permitiría evaluar si ha habido adelantos en la consecución de metas particulares, o si se han emprendido medidas apropiadas para lograr estas metas. Ello equivaldría a incorporar en la evaluación del desempeño un componente orientado a examinar el logro de resultados determinados.

Ya que suele haber un lapso prolongado entre una intervención determinada y los cambios observables como resultado de ella, hay quienes argumentan que la evaluación del desempeño no debe basarse en los resultados exclusivamente, sino también en los procesos propios de un sistema de salud. De ahí que algunos hayan propuesto incluir cálculos de riesgo como si fuesen resultados intermedios. Pero la duda radica en si son o no adecuadas las distintas categorías que describen el potencial, los procesos y los riesgos a la hora de evaluar si ha habido adelantos, especialmente en los distintos subcomponentes del sistema. Si lo son, otra duda radica en determinar cómo se deben elegir estos indicadores. El U.S. National Research Council sugiere cuatro criterios básicos para hacer esta elección:

- las mediciones deben orientarse a objetivos y resultados específicos

- las mediciones deben ser útiles y fáciles de entender

- los datos deben ser los adecuados para permitir las mediciones

- las mediciones deben ser válidas, confiables y flexibles.

\section{La disponibilidad de recursos o el potencial del sistema}

Si se acepta el marco propuesto en la sección anterior, los indicadores de los recursos y su distribución, así como las mediciones del potencial, constituirían la primera dimensión de la evaluación del desempeño de un sistema de salud. No obstante, la medición del potencial de una manera útil y apro- piada para hacer comparaciones plantea un gran reto en sentido teórico y práctico.

\section{La medición del cumplimiento de las funciones}

Hacen falta indicadores que describan la manera en que los sistemas se organizan a la hora de realizar una serie de funciones interdependientes y necesarias para alcanzar determinadas metas. Encontrar indicadores apropiados en este sentido es particularmente difícil en vista de la continua evolución y redefinición que experimenta este campo, donde aún no se ha alcanzado un gran desarrollo conceptual ni metodológico y que se presta a numerosas interpretaciones.

\section{La función rectora de los ministerios de salud}

La reforma del Estado y el proceso de descentralización han llevado a la necesidad de redefinir las funciones de las instituciones dentro del sistema sanitario, particularmente el papel directivo de los ministerios de salud y las funciones esenciales que competen al Estado en los niveles central, intermedio y local. Actualmente, las responsabilidades del Estado están cambiando como resultado de la creciente separación de las áreas de financiamiento, mancomunación de riesgos y prestación de servicios. Estos cambios exigen una reconfiguración de dichas responsabilidades a manera de fortalecer la función directiva de estas entidades. De ahí que se haya dedicado un gran esfuerzo en nuestra Región a medir el desempeño de las llamadas "funciones esenciales en salud pública" (FESP), es decir, aquellas funciones estatales críticas que deben estar presentes para poder mejorar las prácticas en el campo de la salud pública.

A fin de facilitar el debate, se han definido 11 FESP:

- monitoreo y análisis de la situación de salud

- vigilancia, investigación y control de riesgos y daños en salud pública

- promoción de la salud

- participación social y empoderamiento de los habitantes en materia de salud

- diseño de políticas, planificación y capacidad gerencial para apoyar las actividades en salud pública y la función rectora de las autoridades de salud nacionales

- regulación de la salud pública y cumplimiento de las leyes adoptadas

- evaluación y promoción de un acceso equitativo a los servicios de salud indispensables 
- desarrollo de recursos humanos y adiestramiento en salud pública

- garantía de la calidad de los servicios dirigidos a las personas individualmente y a la población

- investigación, desarrollo y adopción de soluciones innovadoras en el campo de la salud pública

- reducción del impacto de las emergencias y de los desastres sobre la salud

El desarrollo de instrumentos para medir el grado en que las autoridades cumplen su función rectora y las FESP implica definir indicadores y normas, así como las mediciones y submediciones que se usarán para verificar los resultados. También implica determinar la validez, fiabilidad y consonancia de cada indicador.

La medicón del grado en que las autoridades de salud cumplen su función rectora y las FESP no es un mero ejercicio de interés metodológico, sino un paso necesario para mejorar la práctica de la salud pública y establecer buenas normas de funcionamiento y parámetros de referencia para mejorar la calidad de manera sostenida. El proceso también llevará a una mayor transparencia en la práctica y prestará mayor claridad a la generación de conocimientos basados en la observación científica (evidence-based practice).

\section{Metas instrumentales o intermedias}

En su mayor parte, los indicadores del logro de metas intermedias se centran en la forma de proveer servicios de salud a las personas individualmente y a la población en general. Guardan estrecha relación con las prácticas gerenciales y los procesos decisorios que competen a los administradores de los servicios y a las autoridades nacionales, regionales y locales. $\mathrm{Si}$ un sistema de salud se define en los términos más amplios que se describieron en la primera parte de este documento, se presenta la dificultad de definir indicadores de la medida en que las autoridades sanitarias interactúan con otros sectores.

A lo largo del tiempo se han sugerido numerosas metas intermedias, algunas de las cuales se aplican hoy en día en Australia, Canadá y Reino Unido. Entre ellas figuran las siguientes:

- acceso (si los pacientes pueden o no obtener los servicios que necesitan en el lugar donde los necesitan y en un lapso corto)

- efectividad (si los servicios funcionan adecuadamente y de qué manera influyen sobre la salud)

- idoneidad (si la atención es la apropiada a la luz de las necesidades de la población y si cumple con las normas establecidas)
- continuidad (cómo están integrados los servicios en cuanto a su coordinación y a la facilidad con que se puede acudir a ellos)

- sustentabilidad (si el sistema puede proveer una infraestructura humana y material y reponder con medidas innovadoras a las necesidades que vayan surgiendo)

- eficiencia (en términos técnicos, si se obtienen los mejores resultados al menor costo posible)

- competencia (si los conocimientos y habilidades de los prestadores de servicios son los apropiados a la luz de los servicios que ofrecen)

- aceptabilidad (si el sistema de salud cumple a satisfacción las expectativas de los usuarios)

\section{Valores sociales y metas sociales ulteriores}

Resulta evidente que la elección de los indicadores de desempeño no se efectúa en un vacío, sino en un entorno caracterizado por ciertos valores. Un aspecto importante que debe tenerse en cuenta en el futuro es si no hay otros valores que no han sido contemplados adecuadamente en el esquem actual y, de ser así, de qué manera se pueden incorporar. Como se señalaba anteriormente, la solidaridad, la humanidad, la universalidad y la conservación de un ambiente sano han surgido una y otra vez en los debates como ejemplos de valores que se han omitido.

\section{LA EVALUACIÓN Y SU RELACIÓN CON LA ADOPCIÓN DE POLÍTICAS Y EL PROCESO DECISORIO}

\section{Los indicadores del cumplimiento de las funciones del sistema}

La evaluación periódica del desempeño exige que cada gobierno monitoree y evalúe los logros de sus sistemas de salud sobre la base de los grupos de funciones que se han definido, y que identifique políticas y estrategias para mejorar el desempeño. Desde la publicación del ISM se han planteado numerosos interrogantes en torno a las implicaciones del monitoreo para la labor de los ministerios de salud y de la OMS, como organismo proveedor de apoyo técnico.

El marco actual para la evaluación del desempeño de los sistemas de salud ayuda a las autoridades a hacer comparaciones entre países. Para mejorar el desempeño, se torna necesario, entonces, tomar medidas en cada una de las cuatro áreas fundamentales - función rectora, financiamiento, provisión y generación de recursos-, pero actualmente no se dispone de herramientas para medir el 
cumplimieno de cada función a la luz de metas instrumentales definidas por consenso. Habrá que decidir si se debe incluir un número limitado de indicadores instrumentales adicionales que representen la fase mediadora entre la provisión de servicios y el logro de las metas, procurando que tengan suficiente flexibilidad para poder cambiar en el corto plazo según las políticas y decisiones. Surgen, entonces, interrogantes sobre cómo definir y medir estos indicadores.

\section{Implicaciones para los sistemas de información de salud}

Los sistemas de información de salud nacionales y subnacionales no están diseñados de una manera que permita efectuar una evaluación global del desempeño de los sistemas de salud, y la OMS aún carece de la capacidad necesaria para ofrecer apoyo técnico en este sentido. La modificación de los sistemas de información de salud dará lugar a extensas deliberaciones con expertos de los Estados Miembros, a fin de determinar qué conjunto mínimo de indicadores instrumentales e intrínsecos deberá medirse con regularidad, quizá mediante encuestas celebradas a intervalos prudentes.

La existencia actual de millares de indicadores de desempeño impone una gran carga a los sistemas de información de salud de todos los Estados Miembros, pero especialmente a los de los países más pobres. Dificulta, además, el examen y el análisis de los datos. Es necesario encontrar la justa medida entre la cobertura de todos los posibles factores que pueden afectar a los resultados, la capacidad del sisema de generar datos sobre ellos con rapidez, y la capacidad de los administradores de digerir la información obtenida.

\section{Participación de la sociedad civil}

Si los diseñadores de políticas aceptan su función directriz en torno a la salud de la población, tendrán que incrementar su interacción con entidades de financiamiento y con proveedores de atención fuera del sector público. La evaluación del desempeño del sistema de salud implica evaluar lo que contribuye cada parte del sistema e idear maneras de mejorar el funcionamiento del sector privado, por ejemplo, en caso de que no estuviese contribuyendo en la medida esperada. Los gobiernos tendrán que mantener un diálogo sostenido con entidades públicas y privadas, sin y con fines de lucro, así como con sus colaboradores en sectores ajenos al de la salud.

\section{El apoyo técnico de la Organización Mundial de la Salud}

La OMS se ve en la necesidad de fortalecer su propia capacidad para ofrecer apoyo técnico a los países en su tarea de evaluar el desempeño de sus sistemas de salud. Esto se logrará mediante extensos diálogos con los gobieros de los países, haciendo hincapié en las siguientes actividades:

- revisar la metodología de evaluación del desempeño empleada en cada país mediante consulta con expertos nacionales

- ayudar a los países que lo soliciten a a) participar en los diálogos sobre las políticas de salud nacionales, b) evaluar las funciones esenciales, c) emprender mediciones y análisis subnacionales, previa revisión de la calidad de los sistemas de información de salud del país para efectuar la evaluación del desempeño del sistema de salud, y d) idear políticas adecuadas para responder a la situación.

\section{SYNOPSIS}

\section{Regional Consultation of the Americas on Health Systems Performance Assessment}

This piece summarizes a document entitled "Critical Issues in Health System Performance Assessment," which was prepared for the Regional Consultation of the Americas on Health Systems Performance Assessment, a meeting held in Washington, D.C., 8 to 10 May 2001. That gathering came about in response to the publication, by the World Health Organization (WHO), of its World Health Report 2000. Done by an advisory group, the Report focused on a comparative evaluation of the performance of the health systems of the WHO Member States. The task of preparing the Report was hindered by a series of factors, from the scarcity of basic data in some countries to the lack of reliable indicators-and especially uniform ones-that would make it possible to draw general conclusions. With a view to overcoming these deficiencies, an innovative methodology was conceived for the Report, based on the evaluation of processes and outcomes, which would make it possible to make valid comparative analyses. Unfortunately, the suitability of the methodology that was used in the Report has been criticized internationally and has led the WHO Director-General to take concrete steps for the next Report, which will come out in October 2002, in order to have improved results. This piece describes measures that need to be taken conceptually and methodologically, with an emphasis on changes in the system for evaluating health system performance and on involving every WHO Member State in the analysis of the data. 